\title{
MHD AND ROTATION EFFECTS ON FLOW PAST AN ACCELERATED VERTICAL PLATE WITH VARIABLE TEMPERATURE AND MASS DIFFUSION IN THE PRESENCE OF CHEMICAL REACTION
}

\author{
R. MUTHUCUMARASWAMY* \\ Department of Applied Mathematics \\ Sri Venkateswara College of Engineering \\ Sriperumbudur 602 105, INDIA \\ E-mail: msamy@svce.ac.in \\ N. DHANASEKAR \\ Department of Mathematics, Mohamed Sathak \\ A.J. College of Engineering \\ Chennai 603 103, INDIA \\ G. EASWARA PRASAD \\ Department of Mathematics, S.T. Hindu College \\ Nagercoil, INDIA
}

\begin{abstract}
An exact analysis of rotation effects on an unsteady flow of an incompressible and electrically conducting fluid past a uniformly accelerated infinite isothermal vertical plate, under the action of a transversely applied magnetic field is presented. The plate temperature is raised linearly with time and the concentration level near the plate is also raised to $C{ }_{w}$. The dimensionless governing equations are solved using the Laplace-transform technique. The velocity profiles, temperature and concentration are studied for different physical parameters such as the magnetic field parameter, chemical reaction parameter, thermal Grashof number, mass Grashof number, Schmidt number, Prandtl number and time. It is observed that the velocity increases with increasing values of the thermal Grashof number or mass Grashof number. It is also observed that the velocity increases with decreasing values of the magnetic field parameter or rotation parameter $\Omega$.
\end{abstract}

Key words: rotation, accelerated, isothermal, vertical plate, heat transfer, diffusion, magnetic field, chemical reaction.

\section{Introduction}

The influence of the magnetic field on a viscous incompressible flow of an electrically conducting fluid has its importance in many applications such as extrusion of plastics in the manufacture of rayon and nylon, purification of crude oil, pulp, paper industry, textile industry and in different geophysical cases etc. In many process industries, the cooling of threads or sheets of some polymer materials is of importance in the production line. The rate of cooling can be controlled effectively to achieve final products of desired characteristics by drawing threads, etc. in the presence of an electrically conducting fluid subject to a magnetic field.

Chemical reactions can be codified as either heterogeneous or homogeneous processes. This depends on whether they occur at an interface or as a single phase volume reaction. In well-mixed systems, the reaction is heterogeneous, if it takes place at an interface and homogeneous, if it takes place in solution. In

\footnotetext{
* To whom correspondence should be addressed
} 
most cases of chemical reactions, the reaction rate depends on the concentration of the species itself. A reaction is said to be of first order, if the rate of reaction is directly proportional to the concentration itself.

Chambre and Young (1958) analyzed a first order chemical reaction in the neighborhood of a horizontal plate. Das et al. (1994) studied the effect of a homogeneous first order chemical reaction on the flow past an impulsively started infinite vertical plate with uniform heat flux and mass transfer. Again, mass transfer effects on a moving isothermal vertical plate in the presence of a chemical reaction was studied by Das et al. (1999). The dimensionless governing equations were solved by the usual Laplace-transform technique.

Gupta et al. (1971) studied free convection of a flow past a linearly accelerated vertical plate in the presence of viscous dissipative heat using the perturbation method. Kafousias and Raptis (1981) extended the above problem to include mass transfer effects subjected to variable suction or injection. Free convection effects on flow past an accelerated vertical plate with variable suction and a uniform heat flux in the presence of a magnetic field was studied by Raptis et al. (1981).

MHD effects on flow past an infinite vertical plate for both the classes of impulse as well as accelerated motion of the plate were studied by Raptis and Singh (1985). Mass transfer effects on flow past a uniformly accelerated vertical plate were studied by Soundalgekar (1982). Basant and Prasad (1990) analyzed mass transfer effects on the flow past an accelerated infinite vertical plate with heat sources. Singh (1984) studied an MHD flow past an impulsively started vertical plate in a rotating fluid. Rotation effects on a hydromagnetic free convective flow past an accelerated isothermal vertical plate were studied by Raptis and Singh (1985). Recently, rotation effects on flow past a vertical plate in the presence of thermal radiation were analyzed by Vijayalakshmi (2009).

Hence, it is proposed to study the effects of rotation on the hydromagnetic free-convection flow of an incompressible viscous and electrically conducting fluid past a uniformly accelerated infinite vertical plate with variable temperature and uniform mass diffusion, in the presence of a chemical reaction of first order. The dimensionless governing equations are solved using the Laplace-transform technique. The solutions are in terms of exponential and complementary error function. Such a study is found useful in magnetic control of molten iron flow in the steel industry, liquid metal cooling in nuclear reactors, magnetic suppression of molten semi-conducting materials and meteorology.

\section{Governing equations}

Consider the unsteady hydromagnetic flow of an electrically conducting incompressible and viscous fluid past a uniformly accelerated motion of an infinite vertical plate when the fluid and the plate rotate as a rigid body with a uniform angular velocity $\Omega^{\prime}$ about the $z^{\prime}$-axis in the presence of an imposed uniform magnetic field $B_{0}$ normal to the plate. Initially, the temperature of the plate and concentration near the plate are assumed to be $T_{\infty}$ and $C_{\infty}^{\prime}$. At time $t^{\prime}>0$, the plate starts moving with a velocity $u=u_{0} t^{\prime}$ in its own plane and the temperature from the plate is raised linearly with time and the concentration level near the plate are also raised to $C^{\prime}{ }_{w}$. Since the plate occupying the plane $z^{\prime}=0$ is of infinite extent, all the physical quantities depend only on $z^{\prime}$ and $t^{\prime}$. It is assumed that the induced magnetic field is negligible so that $\boldsymbol{B}=\left(0,0, B_{0}\right)$. It is also assumed that the effect of viscous dissipation is negligible in the energy equation and there is a first order chemical reaction between the diffusing species and the fluid. Then the unsteady flow is governed by free-convective flow of an electrically conducting fluid in a rotating system under the usual Boussinesq's approximation in a dimensionless form

$$
\begin{aligned}
& \frac{\partial u}{\partial t^{\prime}}-2 \Omega^{\prime} V^{\prime}=g\left(\beta T-T_{\infty}\right)+g \beta^{*}\left(C^{\prime}-C_{\infty}^{\prime}\right)+v \frac{\partial^{2} u}{\partial z^{2}}-\frac{\sigma B_{0}{ }^{2}}{\rho} u \\
& \frac{\partial V^{\prime}}{\partial t^{\prime}}+2 \Omega^{\prime} u=\frac{\partial^{2} V^{\prime}}{\partial z^{2}}-\frac{\sigma B_{0}{ }^{2}}{\rho} V^{\prime},
\end{aligned}
$$




$$
\begin{aligned}
& \rho C_{p} \frac{\partial T}{\partial t^{\prime}}=k \frac{\partial^{2} T}{\partial z^{2}}, \\
& \rho C_{p} \frac{\partial C^{\prime}}{\partial t^{\prime}}=D \frac{\partial^{2} C^{\prime}}{\partial z^{2}}-K_{l}\left(C^{\prime}-C_{\infty}^{\prime}\right),
\end{aligned}
$$

with the following initial and boundary conditions

$$
\begin{array}{lllll}
u=0, & T=T_{\infty}, & C^{\prime}=C_{\infty}^{\prime} \quad \text { for all } & y, t^{\prime} \leq 0, \\
t^{\prime}>0: & u=u_{0} t^{\prime}, & T=T_{\infty}+\left(T_{w}-T_{\infty}\right) A t^{\prime}, & C^{\prime}=C_{w}^{\prime} \quad \text { at } \quad y=0, \\
u \rightarrow 0 & T \rightarrow T_{\infty}, & C^{\prime} \rightarrow C_{\infty}^{\prime} & \text { as } & y \rightarrow \infty .
\end{array}
$$

On introducing the following non-dimensional quantities

$$
\begin{aligned}
U & =\frac{u}{\left(v u_{0}\right)^{1 / 3}}, \quad V=\frac{v}{\left(v u_{0}\right)^{1 / 3}}, \quad t=\left(\frac{u_{0}^{2}}{v}\right)^{\frac{1}{3}}, \quad Z=z\left(\frac{u_{0}}{v^{2}}\right)^{\frac{1}{3}}, \\
\theta & =\frac{T-T_{\infty}}{T_{w}-T_{\infty}}, \quad \mathrm{Gr}=\frac{g \beta\left(T_{w}-T_{\infty}\right)}{u_{0}}, \quad C=\frac{C^{\prime}-C_{\infty}^{\prime}}{C_{w}^{\prime}-C_{\infty}^{\prime}}, \quad \mathrm{Gc}=\frac{g \beta *\left(C_{w}^{\prime}-C_{\infty}^{\prime}\right)}{u_{0}}, \\
M & =\frac{\sigma B_{0}^{2}}{\rho}\left(\frac{v}{u_{0}^{2}}\right)^{1 / 3}, \quad \operatorname{Pr}=\frac{\mu C_{p}}{k}, \quad \mathrm{Sc}=\frac{v}{D}, \quad K=K_{l}\left(\frac{v}{u_{0}^{2}}\right)^{1 / 3} \\
\text { where, } \quad A & \left(\frac{u_{0}^{2}}{v}\right)^{1 / 3},
\end{aligned}
$$

the dimensionless governing equations are as follows

$$
\begin{aligned}
& \frac{\partial U}{\partial t}-2 \Omega V=\operatorname{Gr} \theta+\operatorname{Gc} C+\frac{\partial^{2} U}{\partial Z^{2}}-M U, \\
& \frac{\partial V}{\partial t}+2 \Omega U=\frac{\partial^{2} V}{\partial Z^{2}}-M V, \\
& \frac{\partial \theta}{\partial t}=\frac{1}{\operatorname{Pr}} \frac{\partial^{2} \theta}{\partial Z^{2}}, \\
& \frac{\partial C}{\partial t}=\frac{1}{\operatorname{Sc}} \frac{\partial^{2} C}{\partial Z^{2}}-K C .
\end{aligned}
$$


The initial and boundary conditions in non-dimensional quantities are

$$
\begin{array}{lrrrr}
q=0, & \theta=0, & C=0 & \text { for all } \quad Z, t \leq 0, & \\
t>0: & q=t, & \theta=t, & C=1 \quad \text { at } \quad Z=0, \\
q \rightarrow 0, & \theta \rightarrow 0, & \mathrm{C} \rightarrow 0 \quad \text { as } \quad Z \rightarrow \infty . &
\end{array}
$$

The hydromagnetic rotating free-convection flow past an accelerated vertical plate is described by coupled partial differential Eqs (2.7) to (2.10) with the prescribed boundary conditions (2.11). To solve Eqs (2.7) and (2.8), we introduce a complex velocity $q=U+i V$, Eqs (2.7) and (2.8) can be combined into a single equation

$$
\frac{\partial q}{\partial t}=\operatorname{Gr} \theta+\operatorname{Gc} C+\frac{\partial^{2} q}{\partial Z^{2}}-m q
$$

where,

$$
m=M+2 i \Omega \text {. }
$$

\section{Method of solution}

The dimensionless governing Eqs (2.9), (2.10) and (2.12), subject to the initial and boundary conditions (2.11), are solved by the usual Laplace-transform technique and the solutions are derived as follows

$$
\begin{aligned}
& q=\left(\frac{t}{2}+c+c a t+d\right)\left[\begin{array}{l}
\exp (2 \eta \sqrt{m t}) \operatorname{erfc}(\eta+\sqrt{m t})+ \\
+\exp (-2 \eta \sqrt{m t}) \operatorname{erfc}(\eta-\sqrt{m t})
\end{array}\right]+ \\
& -\eta \sqrt{\frac{t}{m}}\left[\frac{1}{2}+a c\right]\left[\begin{array}{l}
\exp (-2 \eta \sqrt{m t}) \operatorname{erfc}(\eta-\sqrt{m t})+ \\
-\exp (2 \eta \sqrt{m t}) \operatorname{erfc}(\eta+\sqrt{m t})
\end{array}\right]+ \\
& -2 c \operatorname{erfc}(\eta \sqrt{\operatorname{Pr}})-c \exp (a t)\left[\begin{array}{l}
\exp (2 \eta \sqrt{(m+a) t}) \operatorname{erfc}(\eta+\sqrt{(m+a) t})+ \\
+\exp (-2 \eta \sqrt{(m+a) t}) \operatorname{erfc}(\eta-\sqrt{(m+a) t})
\end{array}\right]+ \\
& +c \exp (a t)[\exp (2 \eta \sqrt{a t \operatorname{Pr}}) \operatorname{erfc}(\eta \sqrt{\operatorname{Pr}}+\sqrt{a t})+\exp (-2 \eta \sqrt{a t \operatorname{Pr}}) \operatorname{erfc}(\eta \sqrt{\operatorname{Pr}}-\sqrt{a t})]+ \\
& -d \exp (b t)\left[\begin{array}{c}
\exp (2 \eta \sqrt{(m+b) t}) \operatorname{erfc}(\eta+\sqrt{(m+b) t})+ \\
+\exp (-2 \eta \sqrt{(m+b) t}) \operatorname{erfc}(\eta-\sqrt{(m+b) t})
\end{array}\right]+ \\
& +d \exp (b t)\left[\begin{array}{c}
\exp (-2 \eta \sqrt{\mathrm{Sc}(K+b) t}) \operatorname{erfc}(\eta \sqrt{\mathrm{Sc}}-\sqrt{(K+b) t})+ \\
+\exp (2 \eta \sqrt{\mathrm{Sc}(K+b) t}) \operatorname{erfc}(\eta \sqrt{\mathrm{Sc}}+\sqrt{(K+b) t})
\end{array}\right]+ \\
& -2 a c t\left[\left(1+2 \eta^{2} \operatorname{Pr}\right) \operatorname{erfc}(\eta \sqrt{\operatorname{Pr}})-\frac{2 \eta \sqrt{\operatorname{Pr}}}{\sqrt{\pi}} \exp \left(-\eta^{2} \operatorname{Pr}\right)\right]+ \\
& -d[\exp (2 \eta \sqrt{\operatorname{ScKt}}) \operatorname{erfc}(\eta \sqrt{\mathrm{Sc}}+\sqrt{K t})+\exp (-2 \eta \sqrt{\operatorname{ScKt}}) \operatorname{erfc}(\eta \sqrt{\mathrm{Sc}}-\sqrt{K t})],
\end{aligned}
$$




$$
\begin{aligned}
& \theta=t\left[\left(1+2 \eta^{2} \operatorname{Pr}\right) \operatorname{erfc}(\eta \sqrt{\operatorname{Pr}})-\frac{2 \eta \sqrt{\operatorname{Pr}}}{\sqrt{\pi}} \exp \left(-\eta^{2} \operatorname{Pr}\right)\right], \\
& C=\frac{1}{2}\left[\begin{array}{l}
\exp (2 \eta \sqrt{\operatorname{ScKt}}) \operatorname{erfc}(\eta \sqrt{\operatorname{Sc}}+\sqrt{K t})+ \\
+\exp (-2 \eta \sqrt{\operatorname{Sc} K t}) \operatorname{erfc}(\eta \sqrt{\operatorname{Sc}}-\sqrt{K t})
\end{array}\right]
\end{aligned}
$$

where

$$
\begin{aligned}
& a=\frac{m}{\operatorname{Pr}-1}, \quad b=\frac{m-K \mathrm{Sc}}{\mathrm{Sc}-1}, \quad c=\frac{\mathrm{G}_{\mathrm{r}}}{2 a^{2}(1-\mathrm{Pr})}, \\
& d=\frac{\mathrm{G}_{\mathrm{C}}}{2 b(1-\mathrm{Sc})} \quad \text { and } \quad \eta=\frac{Z}{2 \sqrt{t}} .
\end{aligned}
$$

In order to get the physical insight into the problem, the numerical values of $q$ have been computed from Eq.(2.9). While evaluating this expression, it is observed that the argument of the error function is complex and, hence, we have separated it into real and imaginary parts by using the following formula

$$
\begin{aligned}
& \operatorname{erf}(a+i b)=\operatorname{erf}(a)+\frac{\left(\exp -a^{2}\right)}{2 a \pi}[1-\cos (2 a b)+i \sin (2 a b)]+ \\
& +\frac{2 \exp \left(-a^{2}\right)}{\pi} \sum_{n=1}^{\infty} \frac{\left(\exp -n^{2} / 4\right)}{n^{2}+4 a^{2}}\left[f_{n}(a, b)+i g_{n}(a, b)\right]+\in(a, b)
\end{aligned}
$$

where, $\quad f_{n}=2 a-2 a \cosh (n b) \cos (2 a b)+n \sinh (n b) \sin (2 a b)$.

\section{Results and discussion}

For physical understanding of the problem, numerical computations are carried out for different physical parameters Gr, Gc, Sc, $\operatorname{Pr}, M$ and $t$ upon the nature of the flow and transport. The value of the Schmidt number $\mathrm{Sc}$ is taken to be 0.6 which corresponds to water-vapor. Also, the values of the Prandtl number $\operatorname{Pr}$ are chosen such that they represent air $(\operatorname{Pr}=0.71)$ and water $(\operatorname{Pr}=7.0)$. The numerical values of the velocity, temperature and concentration fields are computed for different physical parameters such as the Prandtl number, rotation parameter, magnetic field parameter, thermal Grashof number, mass Grashof number, Schmidt number and time.

In Fig.1, the temperature profiles are calculated for water and air from Eq.(3.2) and these are shown. The effect of the Prandtl number plays an important role in the temperature field. It is observed that the temperature increases with decreasing the Prandtl number. This shows that heat transfer is greater in air than in water. 


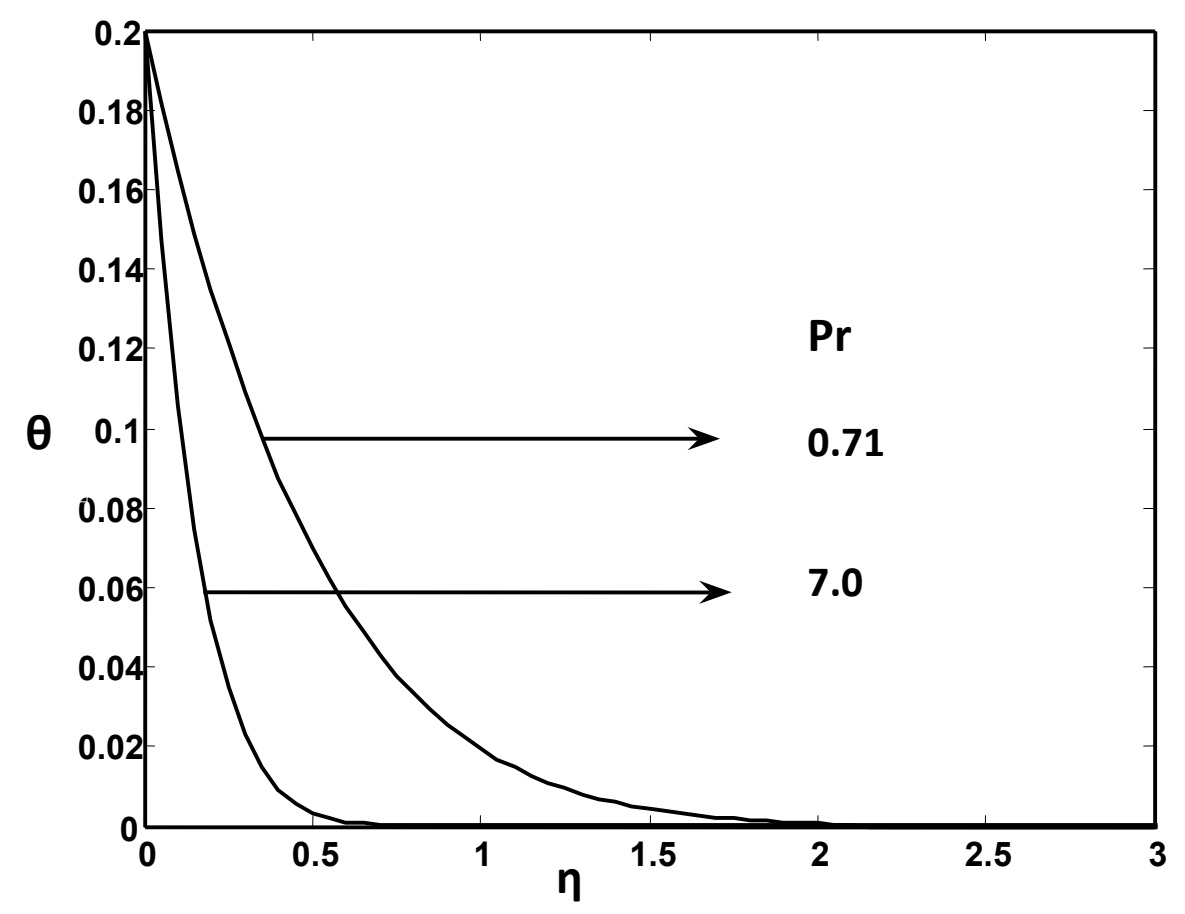

Fig.1. Temperature profiles for different Pr.

Figure 2 represents the effect of concentration profiles for different values of the Schmidt number $(\mathrm{Sc}=0.16,0.3,0.6,2.01), K=0.2$ and $t=0.2$. The effect of concentration is important in the concentration field. The profiles have a common feature that the concentration decreases in a monotone fashion from the surface to a zero value far away in the free stream. It is observed that the wall concentration increases with decreasing values of the Schmidt number.

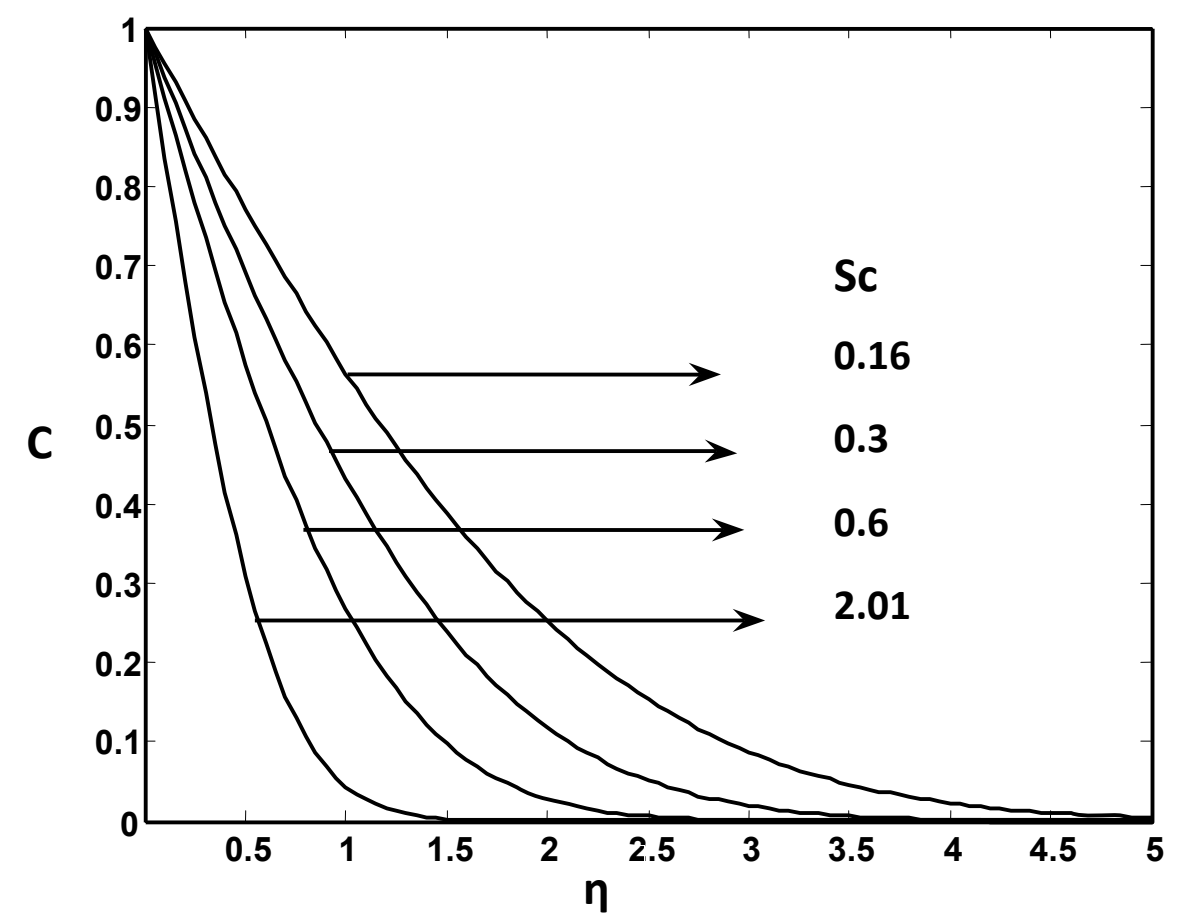

Fig.2. Concentration profiles for different Sc. 
In Fig.3, the concentration fields for different values of the chemical reaction parameter $(K=0.2$, 2,5), $\mathrm{Sc}=0.6$ and $t=0.6$ are shown. The trend shows that the wall concentration increases with decreasing values of the chemical reaction parameter $K$.

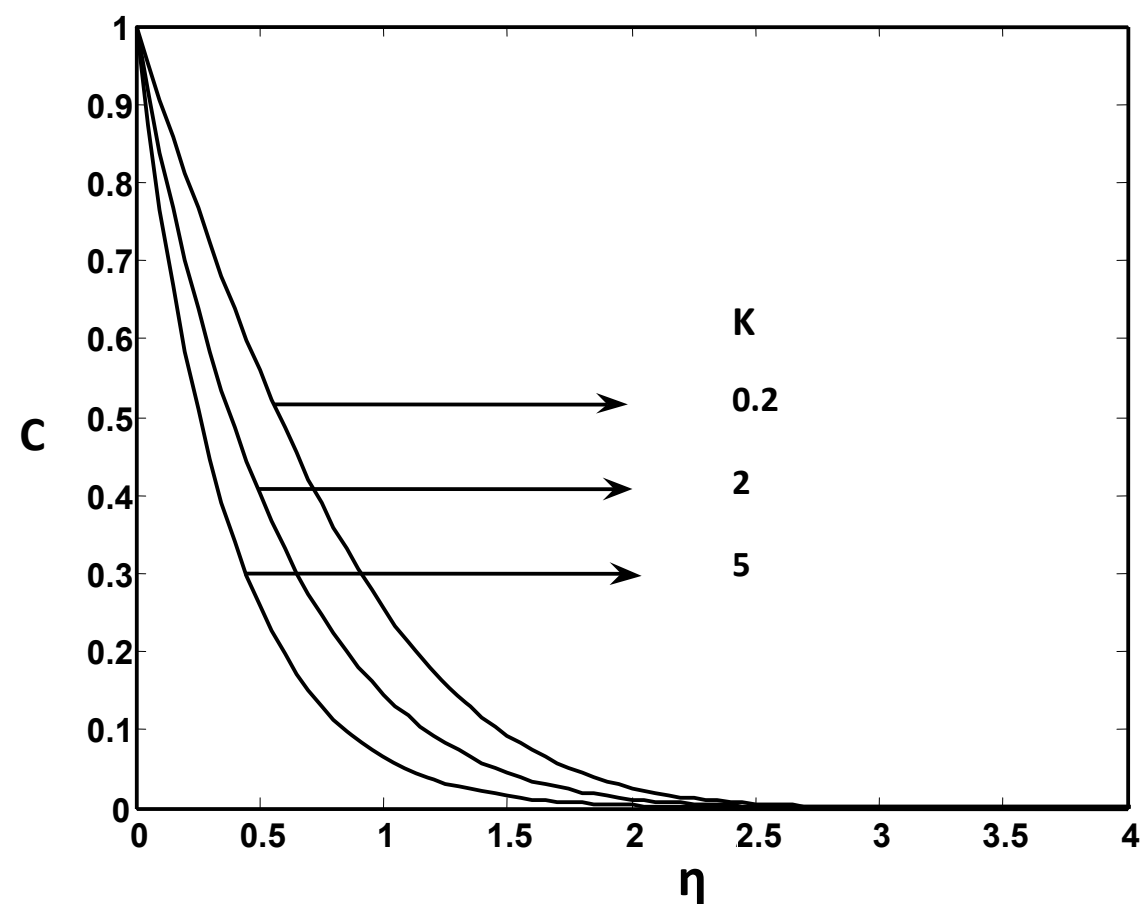

Fig.3. Concentration profiles for different $K$.

Figure 4 illustrates the effects of the magnetic field parameter on the velocity when $(M=1,2,3)$, $\mathrm{Gr}=\mathrm{Gc}=5, \Omega=0.2, \mathrm{Sc}=0.16, K=8, \mathrm{Pr}=7$ and $t=0.6$. It is observed that the velocity increases with decreasing values of the magnetic field parameter. This shows that the increase in the magnetic field parameter leads to a fall in the velocity. This agrees with the expectations, since the magnetic field exerts a retarding force on the free convective flow.

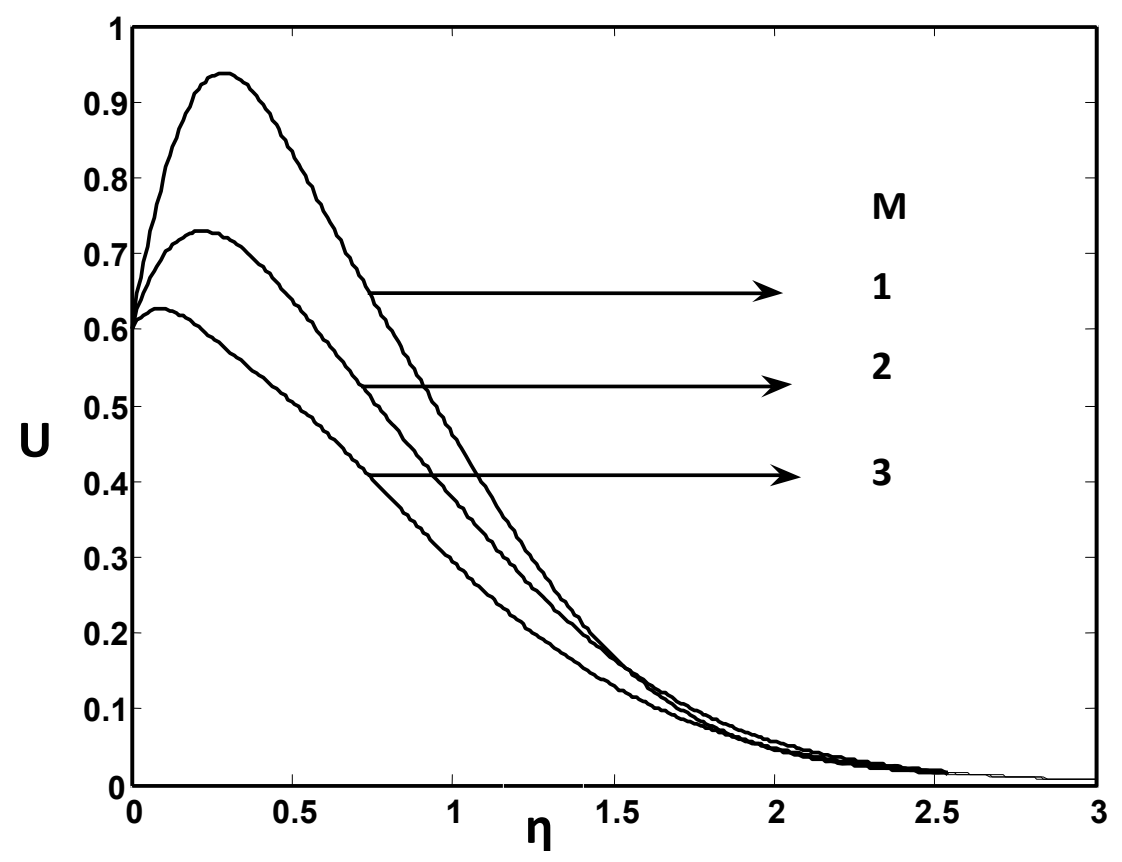

Fig.4. Primary velocity profiles for different $M$. 
Figure 5 demonstrates the effects of different values of the thermal Grashof number $(\mathrm{Gr}=2,5)$, mass Grashof number $(\mathrm{Gc}=2,5), \mathrm{Sc}=0.16, M=5, K=8, \Omega=0.5, \mathrm{Pr}=7$ on the primary velocity at time $t=2.5$. It is observed that the velocity increases with increasing values of the thermal Grashof number or mass Grashof number.

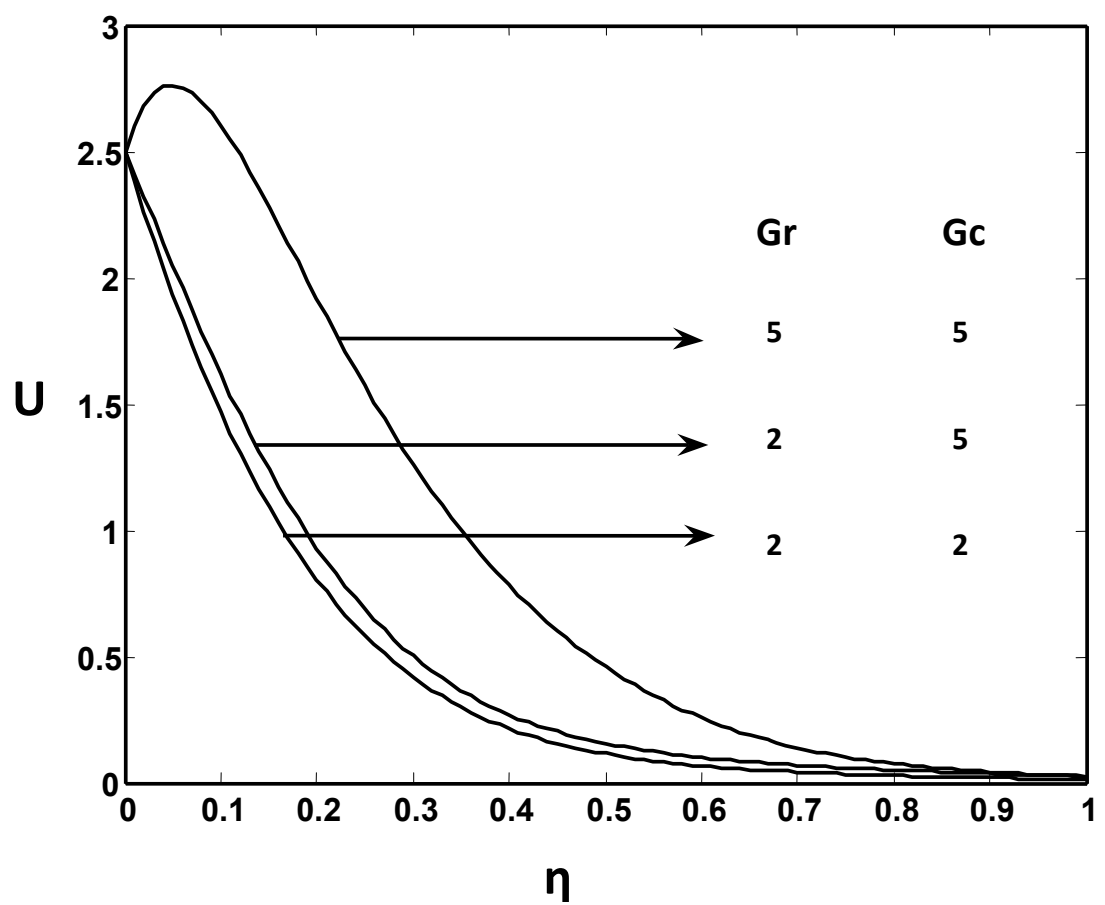

Fig.5. Primary velocity profiles for different $\mathrm{Gr}$ and Gc.

In Fig.6, the secondary velocity profiles for different values of the thermal Grashof number $(\mathrm{Gr}=2,5)$, mass Grashof number $(\mathrm{Gc}=2,5), \Omega=0.2, \mathrm{Sc}=0.8, M=3, \operatorname{Pr}=7$ and $t=0.2$ are presented. The trend shows that the velocity decreases with increasing values of the thermal Grahof number or mass Grashof number.

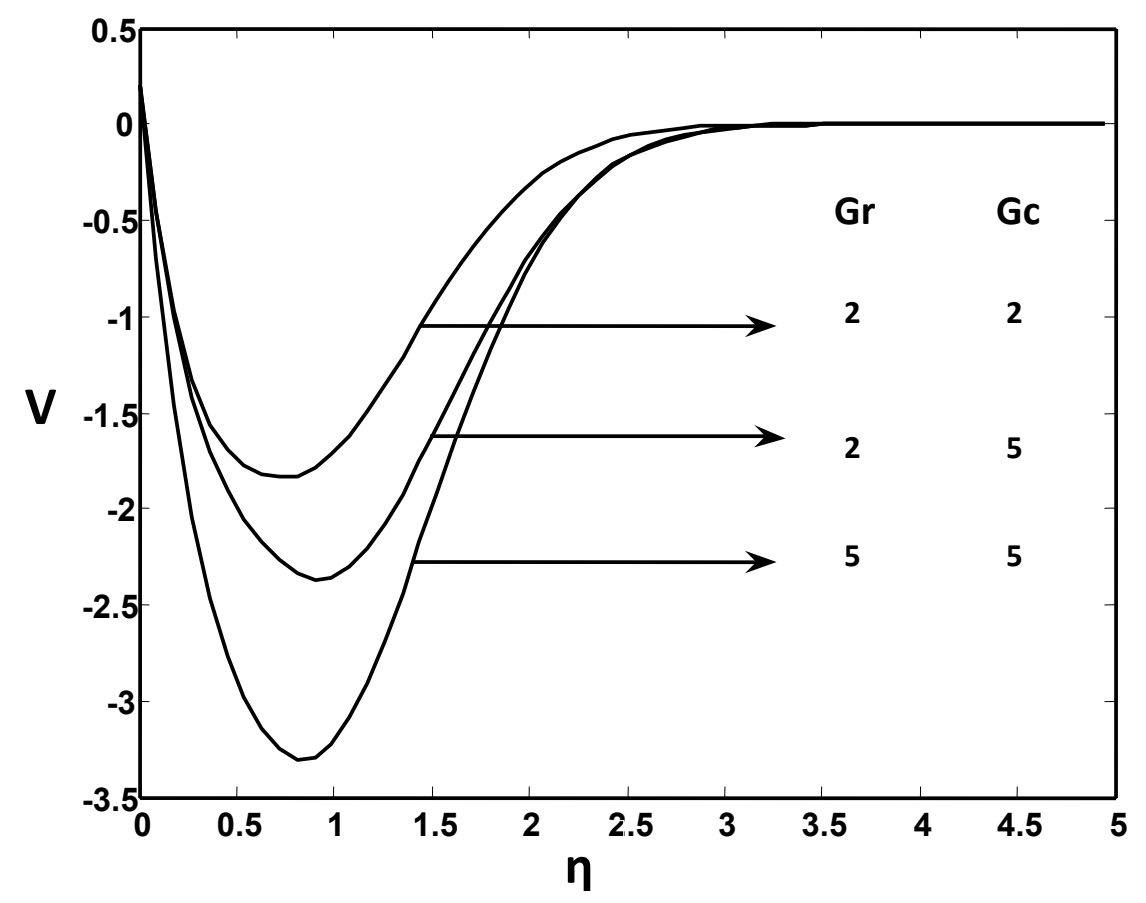

Fig.6. Secondary velocity profiles for different Gr and Gc. 
In Fig.7, the secondary velocity profiles for different rotation parameter $(\Omega=0.5,1), \mathrm{Gr}=\mathrm{Gc}=5, \operatorname{Pr}$ $=7, \mathrm{Sc}=0.8, M=2$ and $t=0.3$ are shown. It is observed that the velocity decreases with increasing values of the rotation parameter $\Omega$.

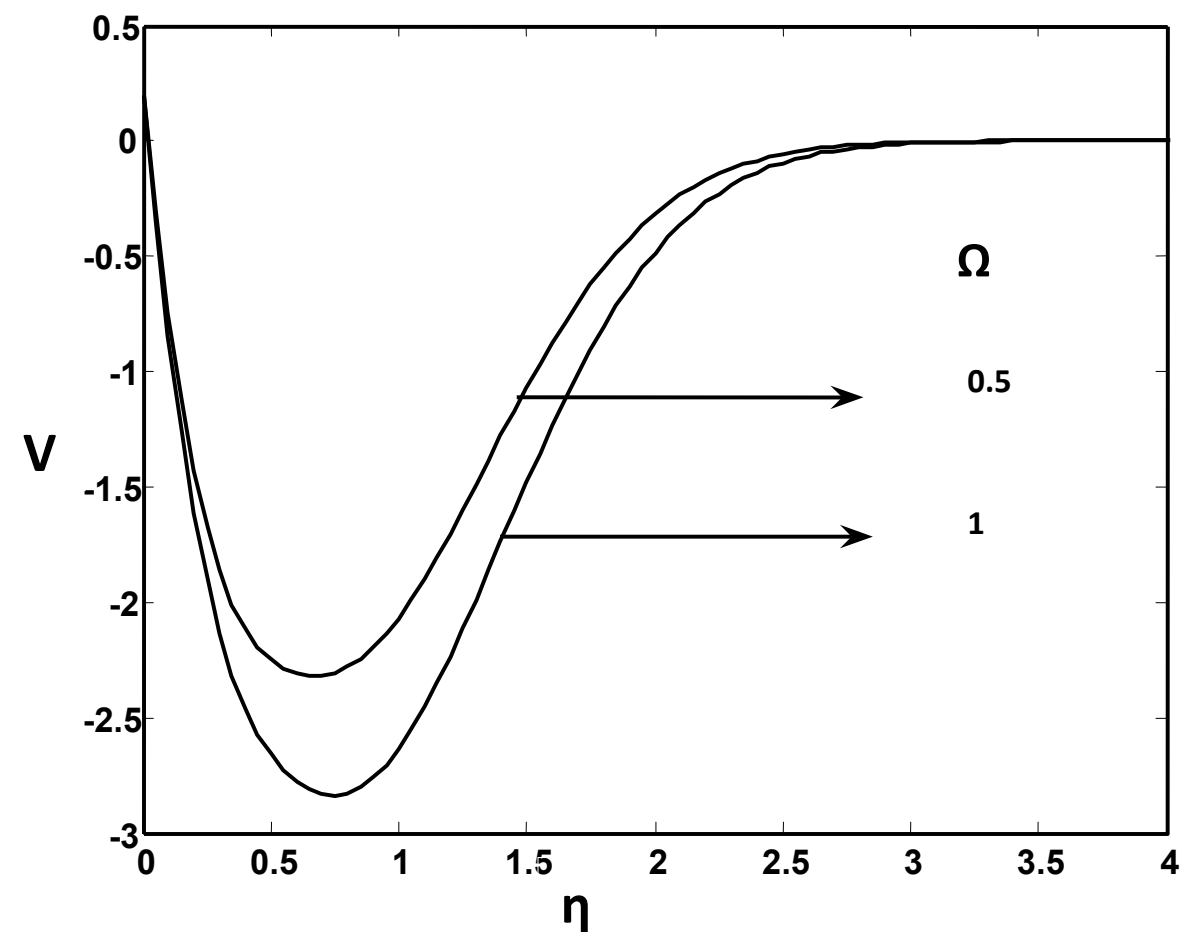

Fig.7. Secondary velocity profiles for different $\Omega$.

\section{Conclusion}

The theoretical solution of flow past a uniformly accelerated infinite isothermal vertical plate in the presence of variable mass diffusion has been studied. The dimensionless governing equations are solved by the usual Laplace transform technique. The effect of different parameters such as the thermal Grashof number, mass Grashof number and $t$ are studied graphically. It is observed that the velocity increases with increasing values of $\mathrm{Gr}, \mathrm{Gc}$ and $t$. But the trend is just reversed with respect to the rotation parameter or magnetic field parameter $M$. It is also observed that the plate concentration decreases with increasing values of the chemical reaction parameter.

\section{Nomenclature}

$$
\begin{aligned}
A & - \text { constant } \\
C & - \text { dimensionless concentration } \\
C^{\prime} & - \text { species concentration in the fluid } \\
C_{p} & - \text { specific heat at constant pressure } \\
C_{w} & - \text { wall concentration } \\
C_{\infty} & - \text { concentration far away from the plate } \\
D & - \text { mass diffusion coefficient } \\
\text { erfc } & - \text { complementary error function } \\
G & - \text { accelerration due to gravity }
\end{aligned}
$$




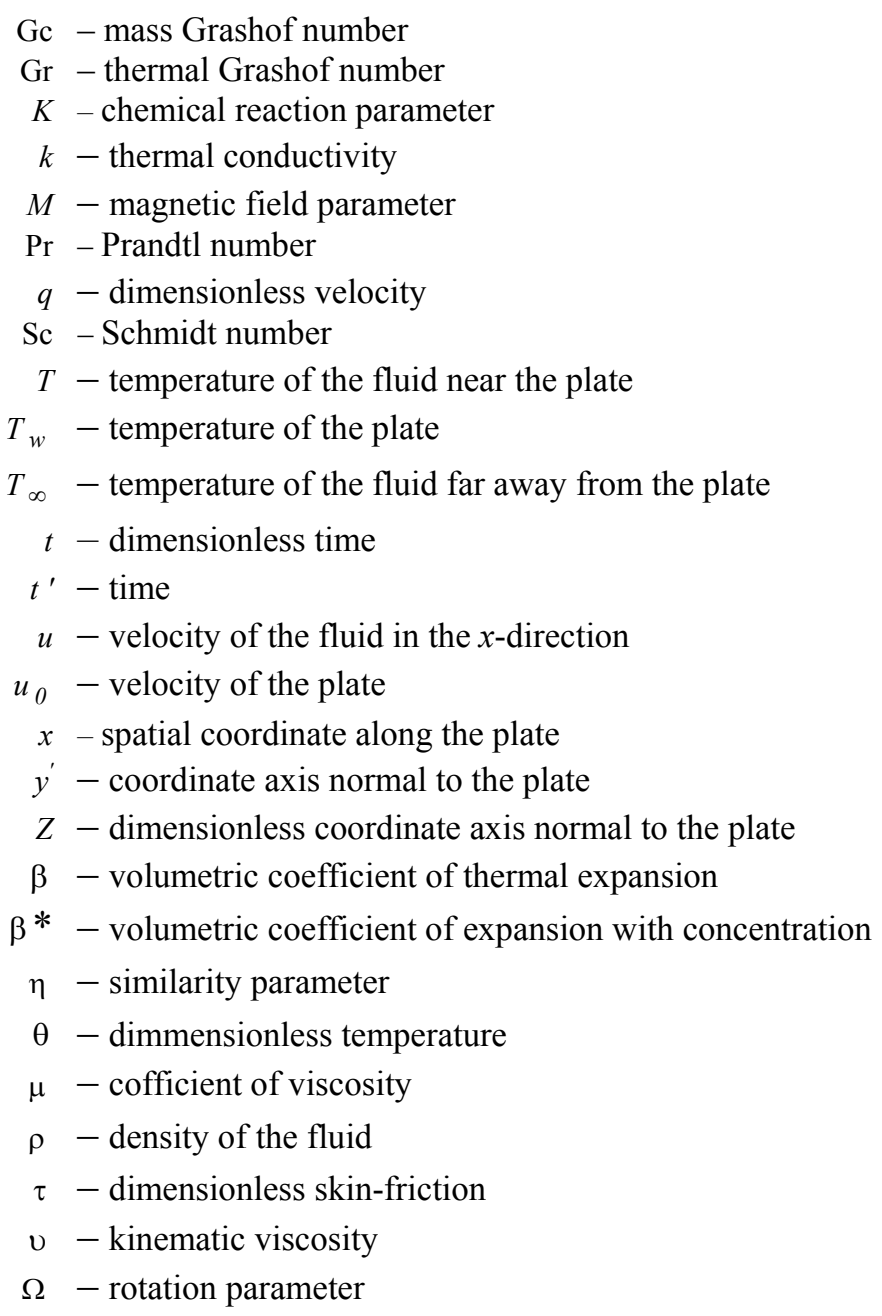

\section{References}

Basanth K.J. and Prasad R. (1990): Free convection and mass transfer effects on the flow past an accelerated vertical plate with heat sources. - Mechanics Research Communications, vol.17, pp.143-148.

Chambre P.L. and Young J.D. (1958): On the diffusion of a chemically reactive species in a laminar boundary layer flow. - The Physics of Fluids, vol.1, pp.48-54.

Das U.N., Deka R.K. and Soundalgekar V.M. (1994): Effects of mass transfer on flow past an impulsively started infinite vertical plate with constant heat flux and chemical reaction. - Forschung im Ingenieurwesen, vol.60, pp.284-287.

Das U.N., Deka R.K. and Soundalgekar V.M. (1999): Effects of mass transfer on flow past an impulsively started infinite vertical plate with chemical reaction. - The Bulletin of Guma, vol.5, pp.13-20.

Gupta A.S., Pop I. and Soundalgekar V.M. (1979): Free convection effects on the flow past an accelerated vertical plate in an incompressible dissipative fluid. - Rev. Roum. Sci. Techn.-Mec. Apl., vol.24, pp.561-568.

Kafousias N.G. and Raptis A. (1981): Mass transfer and free convection effects on the flow past an accelerated vertical infinite plate with variable suction or injection. - Rev. Roum. Sci. Techn.-Mec. Apl., vol.26, pp.11-22.

Raptis A. and Singh A.K. (1981): MHD free convection flow past an accelerated vertical plat. - Letters in Heat and Mass Transfer, vol.8, pp.137-143. 
Raptis A. and Singh A.K. (1985): Rotation effects on MHD free convection flow past an accelerated vertical plate. Mechanics Research Communications, vol.12, pp.31-40.

Raptis A., Tzivanidis G.J. and Peridikis C.P. (1981): Hydromagnetic free convection flow past an accelerated vertical infinite plate with variable suction and heat flux. - Letters in Heat and Mass Transfer, vol.8, pp.137-143.

Singh A.K. (1984): Hydromagnetic free-convection flow past an impulsively started vertical plate in a rotating fluid. International Communications in Heat and Mass Transfer, vol.11, pp.399-406.

Soundalgekar V.M. (1982): Effects of mass transfer on flow past a uniformly accelerated vertical plate. - Letters in Heat and Mass Transfer, vol.9, pp.65-72.

Vijayalakshmi A.R. (2009): Free convection flow past a vertical plate with radiative heat transfer in a rotating medium. - Journal of Intelligent System and Research, vol.3, pp.213-221.

Received: January 18, 2012

Revised: May 8, 2013 